\title{
Erratum
}

\section{Recent Korean isolates of duck hepatitis virus reveal the presence of a new geno- and serotype when compared to duck hepatitis virus type 1 type strains}

\author{
M.-C. Kim ${ }^{1}$, Y.-K. Kwon ${ }^{1}$, S.-J. Joh ${ }^{1}$, S.-J. Kim ${ }^{5}$, C. Tolf ${ }^{2}$, J.-H. Kim ${ }^{4}$, H.-W. Sung ${ }^{3}$,
} A. M. Lindberg ${ }^{2}$, J.-H. Kwon ${ }^{1}$

[Arch Virol (2007) 152: 2059-2072, 10.1007/s00705-007-1023-0]

The authors regret that a sequencing error was made in 3D region of DHV-HS in the above paper. The new sequence of DHV-HS has been updated in GenBank (DQ812094).

In re-analysis of the Simplot, there is no clear signal of recombination between the two strains (DHV-HS and DHV-HSS) so that the SimPlot analysis (Fig. 2) and related paragraphs have become obsolete.

The related paragraphs are as follows:

1. From page 2063 second paragraph (right section) line 21 to page 2064 first paragraph (left section) line 5.

2. On page 2070 third paragraph (left section), from line 14 to line 23 and from 28 to 40 . 